\title{
Autonomic Group Protocol for Distributed Systems
}

\author{
Tomoya Enokido and Makoto Takizawa \\ Department of Computers and Systems Engineering \\ Tokyo Denki University \\ $\{$ eno,taki\}@takilab.k.dendai.ac.jp
}

\begin{abstract}
An autonomic group protocol supports applications with enough quality of service ( $Q o S)$ in change of QoS supported by networks and applications. An autonomic group service is supported for applications by cooperation of multiple autonomous agents. Each agent autonomously takes a class of each protocol function like retransmission. Classes taken by an agent are required to be consistent with but might be different from the others. A group is composed of views each of which is a subset of agents and in each of which agents autonomously take protocol classes consistent with each other. We discuss a model of autonomic group protocol. We also present how to autonomously change retransmission ways in a group as an example.
\end{abstract}

\section{Introduction}

Peer-to-Peer (P2P) systems [1] are getting widely available like grid computing [4] and autonomic computing [5]. Group communication supports basic communication mechanism to realize cooperation of multiple peer processes. Multiple peer processes first establish a group and then messages are exchanged among the processes $[2,7,8,10,12]$. There are group protocols which support the ordered and atomic delivery of messages $[2,7,8,10,12]$. A group protocol is realized by protocol functions; multicast/broadcast, receipt confirmation, detection and retransmission of messages lost, ordering of messages received, and membership management. There are various ways to realize each of these functions like selective and go-back-n retransmissions [6].

The complexity and efficiency of implementation of group protocol depends on what types and quality of service (QoS) are supported by the underlying network. Messages sent by a process may be lost and unexpectedly delayed due to congestions and faults in the network. Thus, QoS parameters are dynamically changed due to congestions and faults. The higher level of communication function is supported, the larger computation and communication overheads are implied. Hence, the system has to take only classes of functions necessary and sufficient to support required service by taking usage of the underlying network service.

The paper [12] discusses a communication architecture which supports a group of multiple processes which satisfies application requirements in change of network service. However, a protocol cannot be dynamically changed each time QoS supported by the underlying network is changed. In addition, each process in a group has to use the same protocol functions.

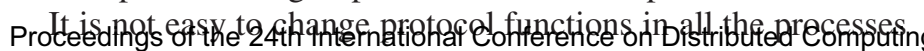

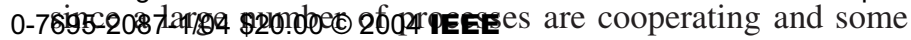
computers like personal computers and mobile computers are not always working well.

In this paper, we discuss an autonomic group protocol which can support types and quality (QoS) of service required by applications even if QoS supported by the underlying network is changed. Each protocol module is realized in an autonomous agent. An agent autonomously changes implementation of each group protocol function depending on network QoS monitored. Here, an agent might take different types of protocol functions from other agents but consistent with the other agents. We discuss what combination of protocol functions are consistent. Each agent has a view which is a subset of agents to which the agent can directly send messages. If a group is too large for each agent to perceive QoS supported by other agents and manage the group membership, the group is decomposed into views. In each view, messages are exchanged by using its own consistent protocol functions. A pair of different views might take different protocols.

In section 2, we show a system model. In section 3, we discuss classes of protocol functions. In section 4 , we present an agent-based architecture to support the autonomic group service. In section 5, we discuss how to change retransmission functions.

\section{System Model}

\subsection{Autonomic group agent}

A group of multiple application processes $A_{1}, \ldots, A_{n}(n$ $\geq 2$ ) are cooperating by taking usage of group communication service. The group communication service is supported by cooperation of multiple peer autonomous group (AG) agents $p_{1}, \ldots, p_{n}$ through exchanging messages by taking usage of underlying network service [Figure 1]. For simplicity, a term "agent" means an AG agent in this paper. The underlying network supports a pair of agents with communication service which is characterized by quality of service (QoS) parameters; delay time [msec], message loss ratio [\%], and bandwidth [bps].

The cooperation of multiple AG agents is coordinated by a group protocol. A group protocol is realized in a collection of protocol functions, transmission, confirmation, retransmission, ordering of message, detection of message lost, coordination schemes, and membership management. There are multiple ways to implement each protocol function. A protocol function class means a way of implementation of protocol function. The classes are stored in a protocol class base (CB). Each application process $A_{i}$ takes group communica-

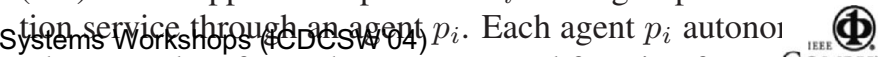
takes one class for each group protocol function from tCOMPUTER tocol class base $\mathrm{CB}$, which can support an applicatio 
necessary and sufficient QoS by taking usage of basic communication service with given QoS supported by the underlying network. Each agent $p_{i}$ monitors QoS supported by the underlying network. The network QoS information monitored is stored in a QoS base (QB) of $p_{i}$. If enough QoS cannot be supported or too much QoS is supported for the application, the agent $p_{i}$ reconstructs a combination of group protocol function classes which are consistent with the other agents by selecting a class for each protocol function in the CB. Here, each agent negotiates with other agents to make a consensus on which class to take for each protocol function. In the paper, we discuss how each agent autonomously change protocol function classes in change of QoS monitored.

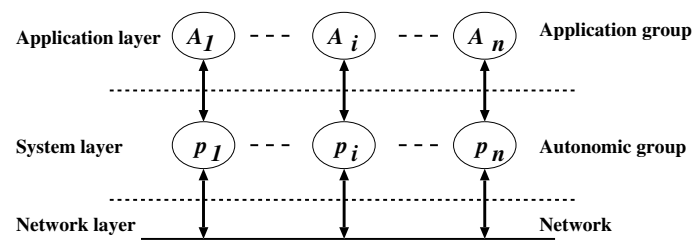

Figure 1. System model.

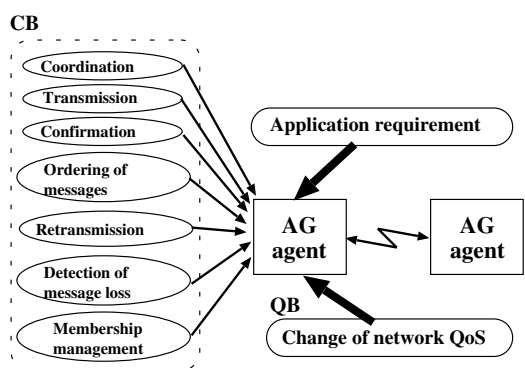

Figure 2. Autonomic group protocol.

\subsection{Views}

A group $G$ is composed of multiple autonomous group (AG) agents $p_{1}, \ldots, p_{n}(n>1)$. An agent is an autonomous peer process which supports application process with group communication service by exchanging messages with other agents. The cooperation of agents is coordinated in a distributed way. In a group $G$ including larger number of agents, it is not easy for each agent to deliver messages to all the agents and maintain membership information. Each agent $p_{i}$ has a view $V\left(p_{i}\right)$ which is a subset of agents to which the agent $p_{i}$ can deliver messages directly or indirectly via agents. Thus, a view is a subgroup of the group $G$. We assume that for every pair of agents $p_{i}$ and $p_{j}, p_{i}$ in $V\left(p_{j}\right)$ iff $p_{j}$ in $V\left(p_{i}\right)$. Each agent $p_{i}$ maintains membership of its view $V\left(p_{i}\right)$. Current information systems are composed of local networks which are interconnected with each other in a trunk network. Here, a view can be a collection of agents interconnected in a local network. A pair of different views $V_{1}$ and $V_{2}$ may include a common agent $p_{k}$. The agent $p_{k}$ is a gateway agent between agents in $V_{1}$ and $V_{2}$. A collection of gateway agents which are interconnected in a trunk network is also a view $V_{3}$. Here, the views $V_{1}, V_{2}$, and $V_{3}$ are hierarchically structured. If an agent $p_{i}$ belongs to only one view, $p_{i}$ is a leaf agent. An agent $p_{i}$ which takes a message $m$ from an application process $A_{i}$ and sends the message $m$ is an original sender agent of the message $m$. If an agent $p_{j}$ delivers a message $m$ to an application process $A_{j}$, the agent $p_{j}$ is an original destination

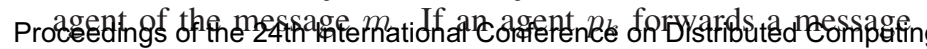
$0-7695$-20870thou \$2G.00 2004meerew $V, p_{k}$ is a routing agent.

Let $\operatorname{src}(m)$ be an original source agent and $\operatorname{dst}(m)$ be a set of original destination agents. A local sender and destination of a message $m$ are agents which send and receive $m$ in a view, respectively.

A view $V$ which includes all the agents in a group $G$ is referred to as complete. A global view is a complete view in a group $G$. If $V \subset G, V$ is partial. A partial view $V$ is changed if an agent joins and leaves the view $V$. If a view $V\left(p_{i}\right)$ is changed, $V\left(p_{i}\right)$ is dynamic. For example, an agent $p_{i}$ sends each message to different agents. If $V\left(p_{i}\right)$ is invariant, $V\left(p_{i}\right)$ is static.

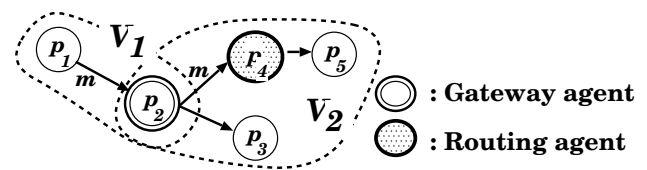

Figure 3. Group views.

\section{Functions of Group Protocol}

A group protocol is realized in a collection of following protocol functions: coordination of the agents, message transmission, receipt confirmation, retransmission, detection of message loss, ordering of messages, and membership management. There are multiple ways to realize each of these functions. A class of a protocol function shows one way to implement the protocol function. One protocol module for an autonomous group (AG) agent is a collection of protocol classes, each of which is for one protocol function. We discuss what classes exist for each protocol function in this section and what combination of classes are consistent in the succeeding section.

There are centralized and distributed approaches to coordinating the cooperation of agents in a view. In the centralized control, there is one centralized controller in a view $V$. On the other hand, there is no centralized controller in the distributed control scheme. Each agent makes a decision on correct receipt, delivery order of messages received, and group membership by itself.
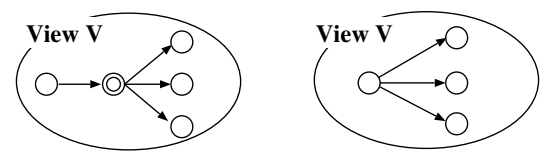

(1) Centralized transmission (2) Direct transmission

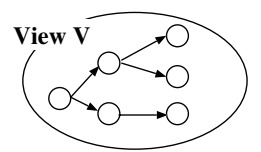

(3) Indirect transmission

\section{Figure 4. Transmission schemes.}

There are centralized, direct, and indirect approaches to multicasting a message to multiple agents in a view [Figure 4]. In the centralized transmission, an agent first sends a message to a forwarder agent and then the forwarder agent forwards the message to all the destination agents in a view [Figure 4 (1)]. The forwarder agent plays a role of a centralized controller. It takes at least two rounds to deliver messages since every message is forwarded by the controller. In the direct transmission, each agent directly not only sends a message to each destination agent but also receives messages from other sender agents in a view $V$ [Figure 4 (2)]. Thus, a

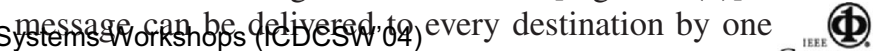
In the indirect transmission, a message is first sent $\mathrm{t} C$ COMPUTER
SOCIETY agent in a view $V$. The agent forwards the message to : 
agent and finally delivers the message to the destination agents in the view $V$ [Figure 4 (3)]. Tree routing [3] is an example. It takes a longer time than one round to deliver a message in the indirect transmission scheme.

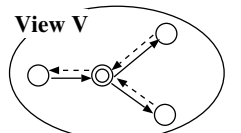

(1) Centralized confirmation

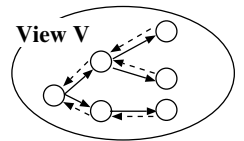

(3) Indirect confirmation

(ation

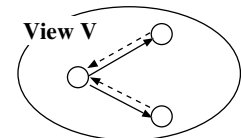

(2) Direct confirmation

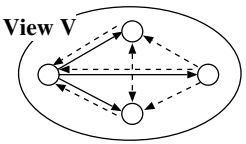

(4) Distributed confirmation
Figure 5. Confirmation schemes.

There are centralized, direct, indirect, and distributed schemes to confirm receipt of a message in a view $V$. In the centralized confirmation, every agent sends a receipt confirmation message to one confirmation agent in a view $V$. After receiving confirmation messages from all the destination agents, the destination agent sends a receipt confirmation to the local sender agent [Figure 5 (1)]. In the direct confirmation, each destination agent $p_{i}$ in the view $V$ sends a receipt confirmation of a message $m$ to the local sender agent $p_{i}$ which first sends the message $m$ in the view $V$ [Figure 5 (2)]. In the indirect confirmation, a receipt confirmation of a message $m$ is sent back to a local sender agent $p_{i}$ in a view $V$ by each agent $p_{j}$ which has received the message $m$ from the local sender agent $p_{i}$ [Figure 5 (3)]. In the distributed confirmation, each agent which has received a message $m$ sends a receipt confirmation of the message $m$ to all the other agents in the same view [10] [Figure 5 (4)]. Each agent in a same view $V$ can know whether or not all the other agents in $V$ have received a same message $m$ by using the distributed confirmation scheme.

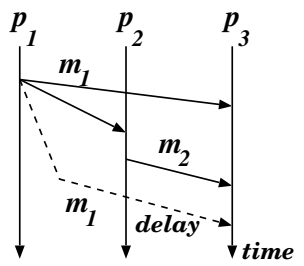

Figure 6. Causally ordered delivery.

A group of multiple agents are exchanging messages in the network. A message $m_{1}$ causally precedes another message $m_{2}\left(m_{1} \rightarrow m_{2}\right)$ if and only if (iff) a sending event of $m_{1}$ happens before a sending event of $m_{2}$ [7]. A message $m_{1}$ is causally concurrent with another message $m_{2}\left(m_{1} \| m_{2}\right)$ if neither $m_{1} \rightarrow m_{2}$ nor $m_{2} \rightarrow m_{1}$. For example, suppose there are three agents $p_{1}, p_{2}$, and $p_{3}$ in a group $G$ [Figure 6]. An agent $p_{1}$ sends a message $m_{1}$ to a pair of agents $p_{2}$ and $p_{3}$. The agent $p_{2}$ sends a message $m_{2}$ to $p_{3}$ after receiving another message $m_{1}$. Here, $m_{1}$ causally precedes $m_{2}\left(m_{1} \rightarrow m_{2}\right)$. Due to communication delay, $m_{1}$ may arrive at $p_{3}$ after $m_{2}$. The agent $p_{3}$ is required to deliver $m_{1}$ before $m_{2}$ because $m_{1}$ $\rightarrow m_{2}$. Messages received are ordered by each agent in the distributed approach. In order to causally deliver messages,

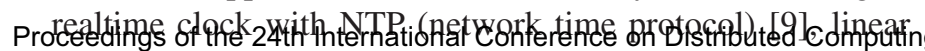

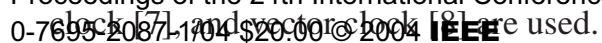

There are sender and destination retransmission schemes with respect to which agent retransmits a message $m$ lost [Figure 7]. Suppose an agent $p_{j}$ sends a message $m$ to agents and one destination agent $p_{i}$ fails to receive $m$. In the sender retransmission, the local sender agent $p_{j}$ which first sent the message $m$ in the view $V$ retransmits the message $m$ to $p_{i}$. In the destination retransmission, one or more than one destination agent in the view $V$ which have safely received the message $m$ forwards $m$ to the agent $p_{i}$ which fails to receive $m$ [Figure 7 (2)]. In the distributed confirmation, each agent can know if every other destination agent safely receives a message $m$.

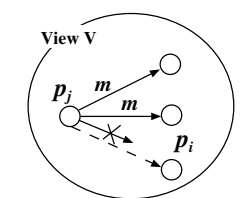

(1) Sender retransmission $\star$ : : fail to receive

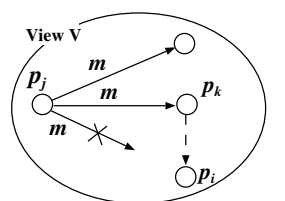

(2) Destination retransmission.

: retransmission

\section{Figure 7. Retransmission scheme.}

There are centralized and distributed ways for managing the membership. In the centralized way, one membership manager communicates with all the member agents to obtain their states. In the distributed way, each agent obtains the states of the other agents by communicating with other agents.

A centralized system is one with centralized coordination, transmission, and confirmation. There is one controller which forwards messages to destination agents and confirms receipt of messages. Most traditional distributed systems like teleconference systems and Amoeba [11] take the centralized approach. A system with distributed coordination, transmission, and centralized confirmation system is classified to be decentralized. ISIS [2] takes the decentralized approach. A sender agent coordinates transmission and receipt of a message. Destination agents send the receipt confirmation to the sender agent. Takizawa et al. [10] take the distributed approach which coordination, transmission, and confirmation are distributed. Here, every destination agent sends the receipt confirmation to not only the sender agent but also all the other destination agents.

\section{Autonomic Group Protocol}

\subsection{Consistent combination of classes}

Each autonomous group (AG) agent takes a collection of classes for protocol functions to communicate with the other agents. In this paper, we consider significant protocol functions, coordination, transmission, confirmation, and retransmission functions. Let $\mathbf{F}$ be a set of the significant protocol functions $\{C$ (coordination), $T$ (transmission), $C F$ (confirmation), $R$ (retransmission) $\}$. For each protocol function $F$ in $\mathbf{F}, C l(F)$ shows a set of classes each of which shows one way of implementation of the protocol function $F$. For example, $C l(C)=\{C$ (centralized $), D($ distributed $)\}$. Table 1 shows classes for protocol functions.

We rewrite $\mathbf{F}$ to be a set $\left\{F_{1}, F_{2}, F_{3}, F_{4}\right\}$ of protocol functions where $\left\langle F_{1}, F_{2}, F_{3}, F_{4}\right\rangle=\langle C, T, C F, R\rangle$. A tuple \langle $\left.c_{1}, c_{2}, c_{3}, c_{4}\right\rangle \in C l\left(F_{1}\right) \times C l\left(F_{2}\right) \times C l\left(F_{3}\right) \times C l\left(F_{4}\right)$ is referred to as a protocol instance. Each agent takes a protocol instance $C=\left\langle c_{1}, c_{2}, c_{3}, c_{4}\right\rangle$, i.e. a class $c_{i}$ is taken for each

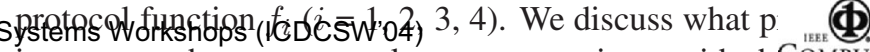
instance each agent can take to communicate with thCOMPUTER agents. 
Table 1. Protocol classes.

\begin{tabular}{|l|l|}
\hline Function $f$ & Protocol classes $C l(f)$ \\
\hline$C$ & $\{C($ centralized $), D($ distributed $)\}$ \\
\hline$C F$ & $\{$ Cen $($ centralized $)$, Dir $($ direct $)$, Ind $($ indirect $)$, Dis $($ distributed $)\}$ \\
\hline$T$ & $\{C($ centralized $), D($ direct $), I($ indirect $)\}$ \\
\hline$R$ & $\{S($ sender $), D($ destination $)\}$ \\
\hline
\end{tabular}

As discussed in the preceding section, the destination retransmission scheme can be taken in the distributed confirmation scheme but not in the centralized one. A protocol instance $\left\langle c_{1}, c_{2}, c_{3}, c_{4}\right\rangle$ is referred to as consistent iff an agent taking the instance can operate. If an agent takes an inconsistent protocol instance, the agent cannot work. Thus, only some protocol instances of function classes are consistent. An agent can take only a consistent protocol instance. Table 2 summarizes possible protocol profiles. A protocol profile is a consistent protocol instance which each agent can take. Protocol profiles are shown in Table 2. A profile signature " $c_{1} c_{2} c_{3} c_{4}$ " denotes a protocol profile $\left\langle c_{1}, c_{2}, c_{3}, c_{4}\right\rangle$. For example, $D D D i r S$ shows a protocol profile $\langle D, D, D i r, S\rangle$ which is composed of distributed control, direct transmission, direct confirmation, and sender retransmission. Let $\mathbf{P}$ be a set of the protocol profiles which are show in Table 2.

\subsection{Consistent set of profiles}

Suppose autonomous group (AG) agents $p_{1}, \ldots, p_{n}$ are in a view $V$ of a group $G$. Let $C_{i}$ show a consistent protocol instance, i.e. protocol profile taken by an agent $p_{i}, C_{i}=\left\langle c_{i 1}, \ldots\right.$, $\left.c_{i 4}\right\rangle \in \mathbf{P}$. A global protocol instance $C$ for a view $V=\left\{p_{1}, \ldots\right.$, $\left.p_{n}\right\}$ is a tuple $\left\langle C_{1}, \ldots, C_{n}\right\rangle$ where each $C_{i}$ is a protocol profile which an agent $p_{i}$ takes. Here, each $C_{i}$ is referred to as local protocol instance of an agent $p_{i}(i=1, \ldots, n)$. In traditional protocols, every agent has to take a same local protocol instance, i.e. $C_{1}=\cdots=C_{n}$. Hence, if some agent $p_{i}$ would like to change a class $c_{i k}$ of a protocol function $F_{k}$ with another one $c_{i k}$, all the agents have to be synchronized to make consensus on a new protocol instance. A global protocol instance $C=\left\langle C_{1}, \ldots, C_{n}\right\rangle$ is referred to as complete if $C_{1}=\cdots=C_{n}$. If $C_{i} \neq C_{j}$ for some pair of agents $p_{i}$ and $p_{j}$, a global protocol instance $C=\left\langle C_{1}, \ldots, C_{n}\right\rangle$ is incomplete. A global protocol instance $C=\left\langle C_{1}, \ldots, C_{n}\right\rangle$ is consistent if a collection of agents where each agent $p_{i}$ takes $C_{i}$ can be cooperating. A global protocol profile is a consistent global protocol instance. It is trivial a complete global protocol instance is consistent. In this paper, we discuss a group protocol where a view of agents $p_{1}, \ldots, p_{n}$ can take an incomplete global protocol instance $C$ $=\left\langle C_{1}, \ldots, C_{n}\right\rangle$. First, suppose that a global protocol instance $C=\left\langle C_{1}, \ldots, C_{m}\right\rangle$ is complete and some agent $p_{i}$ changes a local protocol instance $C_{i}$ with another one $C_{i}^{\prime}$. We discuss whether or not a global protocol instance $\left\langle C_{1}, \ldots, C_{i-1}, C_{i}^{\prime}\right.$, $\left.C_{i+1}, \ldots, C_{n}\right\rangle$ is consistent, i.e. all the agents $p_{1}, \ldots, p_{n}$ can cooperate even if $C_{i}^{\prime} \neq C_{j}$ for some agent $p_{j}$.

According to change of network QoS and application requirement, each agent autonomously changes the protocol profile. For example, suppose an agent $p_{3}$ belongs to a pair of views $V_{1}$ and $V_{2}$ [Figure 8]. In the view $V_{1}$ where all of the agents take DDDirS, an agent $p_{1}$ sends a message $m$ to all the other agents. On receipt of the message $m$, an agent $p_{3}$ with

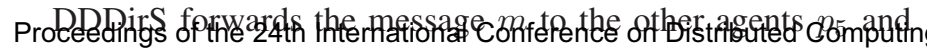

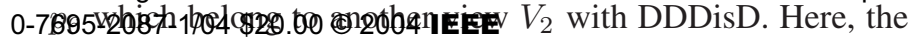
agent $p_{3}$ can receive the receipt confirmation of the message $m$ from a pair of agents $p_{5}$ and $p_{6}$ in the view $V_{2}$. In addition, the agent $p_{3}$ sends back the receipt confirmation of the message $m$ to the original sender agent $p_{1}$. Here, the original sender agent $p_{1}$ can receive the receipt confirmation from all the destination agents in the view $V_{1}$. Therefore, the agent $p_{3}$ does not need to change the profile since the agent $p_{3}$ can forward the message $m$ to another agent in the view $V_{2}$.

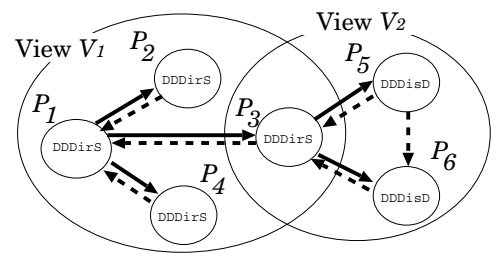

Figure 8. Change of profiles.

\section{Retransmission}

We discuss how an autonomous group (AG) agent can autonomously change the retransmission classes in a group as an example.

\subsection{Cost model}

Suppose there are three autonomic group (AG) agents $p_{s}$, $p_{t}$, and $p_{u}$ in a view $V$. An agent $p_{s}$ sends a message $m$ to a pair of agents $p_{t}$ and $p_{u}$. Then, the agent $p_{t}$ receives the message $m$ while another agent $p_{u}$ fails to receive $m$. Here, $p_{u}$ is referred to as faulty. The following notations are used to discuss a cost model for a pair of agents $p_{s}$ and $p_{t}$ :

1. $d_{s t}=$ delay time between agents $p_{s}$ and $p_{t}$ [msec].

2. $f_{s t}=$ probability that a message is lost.

3. $b_{s t}=$ bandwidth [bps].

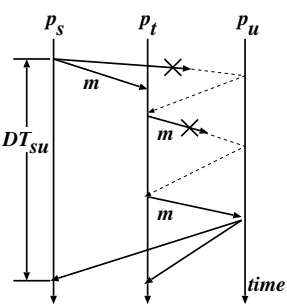

A. $d_{s t} \leq d_{s u}+d_{t u}$

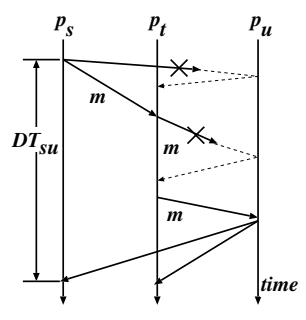

B. $d_{s t} \geq d_{s u}+d_{t u}$

\section{Figure 9. Destination retransmission.}

First, let us consider the sender retransmission. Let $|m|$ show the size of a message $m$ [bit]. It takes $\left(2 d_{s u}+|m| / b_{s u}\right)$ [msec] to detect message loss after the agent $p_{s}$ sends a message $m$. Then, the agent $p_{s}$ retransmits $m$ to $p_{u}$. Here, the message $m$ may be lost again. The expected time $S T_{s u}$ and number $S N_{s u}$ of messages to be transmitted to deliver a message $m$ to a faulty destination $p_{u}$ are given as follows:

1. $S T_{s u}=\left(2 d_{s u}+|m| / b_{s u}\right) /\left(1-f_{s u}\right)$.

2. $S N_{\text {su }}=1 /\left(1-f_{s u}\right)$.

In the destination retransmission, some destinatiorCOMPUTER $p_{t}$ forwards the message $m$ to the agent $p_{u}$ [Figure 9 
Table 2. Protocol profiles.

\begin{tabular}{|l|l|l|l|l|}
\hline Control & Transmission & Confirmation & Retransmission & Signature \\
\hline \hline Centralized & Centralized & Centralized & Sender & CCCenS \\
\hline Distributed & \multirow{2}{*}{ Direct } & Direct & Sender & DDDirS \\
\cline { 3 - 5 } & & Distributed & Sender & DDDisS \\
\cline { 3 - 5 } & & Destination & DDDisD \\
\cline { 3 - 5 } & \multirow{3}{*}{ Indirect } & Direct & Sender & DIDirS \\
\cline { 3 - 5 } & & Indirect & Sender & DIIndS \\
\cline { 3 - 5 } & & Distributed & Sender & DIDisS \\
\cline { 4 - 5 } & & Destination & DIDisD \\
\hline
\end{tabular}

expected time $D T_{s u}$ and number $D N_{s u}$ of messages to deliver a message $m$ to $p_{u}$ are given as follows:

1. $D T_{s u}=\left(d_{s u}+|m| / b_{s u}+d_{u t}\right)+\left(2 d_{u t}+|m| / b_{u t}\right) /(1$ $\left.-f_{u t}\right)$ if $d_{s t} \leq d_{s u}+d_{u t}$.

$D T_{s u}=\left(d_{s t}+|m| / b_{s t}\right)+\left(2 d_{u t}+|m| / b_{u t}\right) /\left(1-f_{u t}\right)$ otherwise.

2. $D N_{s u}=1+1 /\left(1-f_{u t}\right)$.

If $S T_{s u}>D T_{s u}$, the destination agent $p_{t}$ can forward the message $m$ to the faulty agent $p_{u}$ because the message $m$ lost can be delivered earlier.

Each agent $p_{t}$ monitors delay time $d_{u t}$, bandwidth $b_{u t}$, and message loss probability $f_{u t}$ for each agent $p_{u}$ which are received in the QoS base (QB). For example, the agent $p_{t}$ obtains the QoS information by periodically sending QoS information messages to all the agents in a view. The agent $p_{t}$ maintains the quality of service (QoS) information in a variable $Q$ of $\mathrm{QB}$ where $Q_{u t}=\left\langle b_{u t}, d_{u t}, f_{u t}\right\rangle$ for $u=1, \ldots, n$. If the agent $p_{t}$ receives QoS information from another agent $p_{s}, Q_{s u}=\left\langle b_{s u}\right.$, $\left.d_{s u}, f_{s u}\right\rangle$ for $u=1, \ldots, n$.

\subsection{Change of retransmission scheme}

Suppose an agent $p_{s}$ sends a message $m$ and every agent $p_{t}$ take the sender retransmission scheme, $C_{t}=\langle\cdots, S\rangle$. As shown in Figure 10, an agent $p_{u}$ fails to receive the message $m$. According to the change of QoS supported by the underlying network, the sender agent $p_{s}$ makes a decision to change the retransmission scheme with the destination one, say an agent $p_{t}$ forwards the message $m$ to the agent $p_{u}$. However, the agent $p_{t}$ still takes the sender retransmission. Here, no agent forwards the message $m$ to $p_{u}$.

Next, suppose all the agents is taking the destination retransmission scheme. Here, QoS supported by the network is changed and the agent $p_{t}$ decides to take the sender retransmission scheme. However, no agent forwards the message $m$ to the agent $p_{u}$ since the sender agent $p_{s}$ still takes the destination retransmission scheme. In order to prevent these silent situations, we take a following protocol:

1. A sender agent $p_{s}$ sends a message $m$ to all the destination agents. Every destination agent sends receipt confirmation not only to the sender agent $p_{s}$ but also to the other destination agents [Figure 10].

2. If an agent $p_{t}$ detects that a destination agent $p_{u}$ has not received the message $m, p_{t}$ selects a retransmission scheme which $p_{t}$ considers to be optimal based on the QoS information $Q$.
2.2 If $p_{t}$ is a sender of a message $m$ and takes a sender retransmission scheme, $p_{t}$ retransmits $m$ to $p_{u}$. If $p_{t}$ takes a destination retransmission scheme, $p_{t}$ waits for Retx message from a destination. If $p_{t}$ does not receive Retx, $p_{t}$ retransmits $m$ to $p_{u}$.

It is straightforward for the following theorem to hold from the definition.

[Theorem] At least one agent forwards a message $m$ to an agent which fails to receive the message $m$.

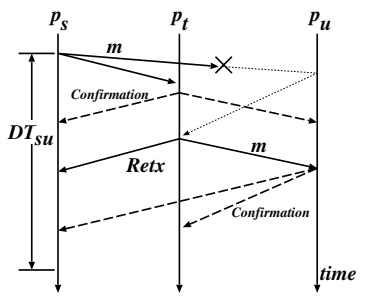

Figure 10. Retransmission.

\section{Evaluation}

We evaluate the autonomic group protocol (AGP) in terms of delivery time of a lost message. We make the following assumptions on this evaluation.

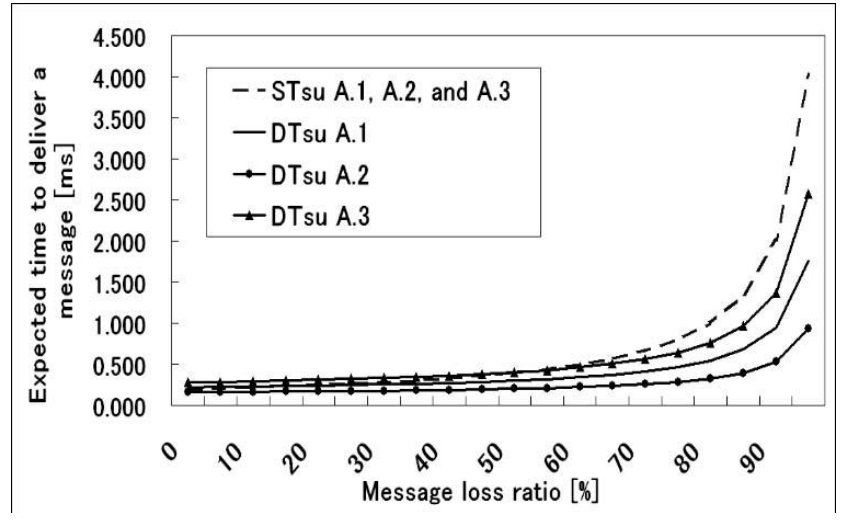

Figure 11. $d_{s u} \geq d_{s t}+d_{u t}$.

1. $d_{s t}=d_{t s}$ for every pair of $p_{s}$ and $p_{t}$.

2. The protocol processing time of every process is same.

3. No confirmation message is lost although messages may be lost.

Let us consider a view $V=\left\{p_{s}, p_{t}, p_{u}\right\}$ where every agent takes a profile DDDisS, distributed control, direct transmission, distributed confirmation, and sender retransmission. 


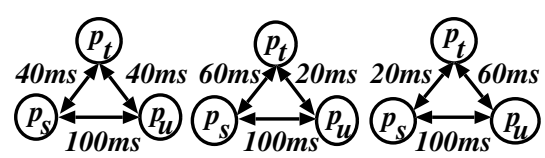

$\begin{array}{lll}\text { 1. } d s t=d t u & \text { 2. } d s t>d t u & \text { 3. } d s t<d t u\end{array}$

A. $d s u \geq d s t+d t u$

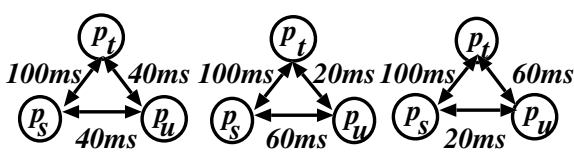

1. $d s u=d t u$

B. $d s u \leq d s t+d t u, d s t>d s u$ and $d s t>d t u$

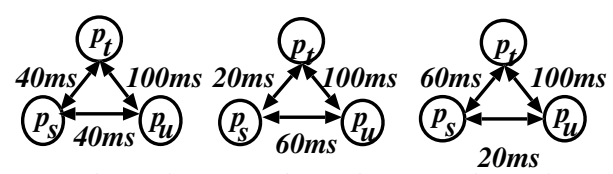

$\begin{array}{ll}\text { 1. } d s u=d s t & \text { 2. } d s u>d s t\end{array}$

3. $d s u<d s t$

C. $d s u \leq d s t+d t u, d t u>d s u$ and $d t u>d s t$

Figure 12. AG agent graph.

the sender $p_{s}$ and destination $p_{t}$ detect the destination agent $p_{u}$ fails to receive the message $m$, the agents $p_{s}$ and $p_{t}$ autonomously select a retransmission scheme based on the QoS information. Here, we evaluate time to deliver a message $m$ to a faulty agent $p_{u}$. In the view $V$, we assume that bandwidth between every pair of agents is same $\left(b_{s t}=b_{s u}=b_{u t}=10 \mathrm{Mbps}\right)$ and $f_{s t}=f_{s u}$ and $f_{u t}=0 \%$. Figure 12 shows an AG agent graph for the view $V$ where each node denotes an agent and each edge shows a communication channel between agents. A label of the edge indicates delay time.

First, we consider a case $d_{s u} \geq d_{s t}+d_{u t}$. There are further cases: $d_{s t}=d_{u t}$ [Figure 12 A.1], $d_{s t}>d_{u t}$ [Figure 12 A.2], and $d_{s t}<d_{u t}$ [Figure 12 A.3]. Figure 11 shows the expected time $D T_{s u}$ for three cases. In Figure 11, horizontal axis shows a message loss probability of $f_{s u}$ and $f_{u t}$. For case of Figure 12 A.2, $D T_{s u}<S T_{s u}$. For case of Figure 12 A.1, $D T_{s u}<$ $S T_{s u}$ if $f_{s u}>15 \%$ and $f_{u t}>15 \%$. For case of Figure 12 A.3, $D T_{s u}<S T_{s u}$ if $f_{s u}>50 \%$ and $f_{u t}>50 \%$.

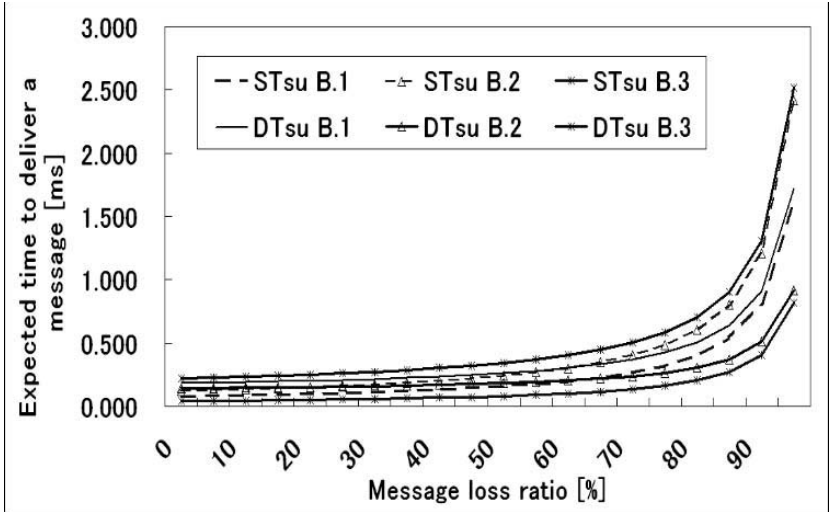

Figure 13. $d_{s u} \leq d_{s t}+d_{u t}, d_{s t}>d_{s u}$, and $d_{s t}>d_{u t}$.

Next, we consider a case $d_{s u} \leq d_{s t}+d_{u t}$. There are further following cases [Figure 12]:

a. $d_{s t}>d_{s u}$ and $d_{s t}>d_{u t}: d_{s u}=d_{u t}\left[\right.$ B.1], $d_{s u}>d_{u t}$ [B.2], and $d_{s u}<d_{u t}$ [B.3].

b. $d_{u t}>d_{s u}$ and $d_{u t}>d_{s t}: d_{s u}=d_{s t}$ [C.1], $d_{s u}>d_{s t}$ [C.2], and $d_{s u}<d_{s t}[\mathrm{C} .3]$.

The expected time $D T_{s u}$ [Figure $12 \mathrm{~B}$ and $12 \mathrm{C}$ ] is shown for these six cases in Figures 13 and 14. For cases of Figure 12 B.1 and B.3, $D T_{s u}>S T_{s u}$. For case of Figure 12 B.2, $D T_{s u}$ $<S T_{s u}$ if $f_{s u}>20 \%$ and $f_{u t}>20 \%$. For case of Figure 12 $\mathrm{C}, D T_{s u}>S T_{s u}$.

\section{Concluding Remarks}

In this paper, we discussed an agent-based architecture to support distributed applications with autonomic group service in change of network and application QoS. Autonomous group

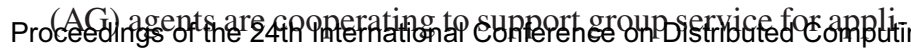
$0-7696$ - 208 W in group communication protocols. Every agent autonomously

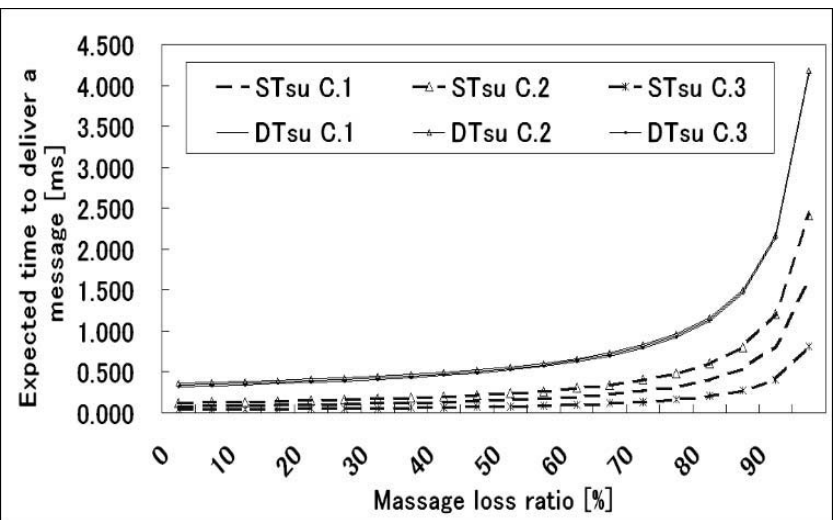

Figure 14. $d_{s u} \leq d_{s t}+d_{u t}, d_{u t}>d_{s u}$, and $d_{u t}>d_{s t}$.

changes class of each protocol function which may not be the same as but are consistent with the other agents in a group. We discussed how to support applications with the autonomic group service by changing retransmission schemes, sender and destination retransmission as an example. We showed which retransmission scheme can be adopted for types of network configuration in the evaluation.

\section{References}

[1] P. E. Agre. P2p and the promise of internet equality. Communication of the ACM, 46(2):39-42, 2003.

[2] S. A. Birman, K. and S. P. Lightweight causal and atomic group multicast. ACM Trans. on Computer Systems, 9(3):272-290, 1991.

[3] S. Deering. Host groups: A multicast extension to the internet protocol. RFC 966, 1985.

[4] I. Foster and C. Kesselman. The Grid: Blueprint for a New Computing Infrastructure. Morgan Kaufmann Publishers, 1999.

[5] IBM Corporation. Autonomic computing architecture : A blueprint for managing complex computing environments. 2002. http://www-3.ibm.com/autonomic/pdfs/ACwhitepaper 1022.pdf.

[6] M. F. Kaashoek and A. S. Tanenbaum. An evaluation of the amoeba group communication system. Proc. of IEEE ICDCS16, pages 436-447, 1996.

[7] L. Lamport. Time, clocks, and the ordering of events in a distributed system. CACM, 21(7):558-565, 1978.

[8] F. Mattern. Virtual time and global states of distributed systems. Parallel and Distributed Algorithms, pages 215-226, 1989.

[9] D. L. Mills. Network time protocol. RFC 1305, 1992.

[10] A. Nakamura and M. Takizawa. Reliable broadcast protocol for selectively ordering pdus. Proc. of IEEE ICDCS-11, pages 239-246, 1991.

[11] C. Steketee, W. P. Zhu, and P. Moseley. Implementation of process migration in amoeba. Proc. of IEEE ICDCS-14, pages 194-201, 1994.

Systam RWwank group communication system. CACM, 39(4):76-83, 19 COMPUTER 